\title{
Diagnostic cardiac catheterisation in a hospital without on-site cardiac surgery
}

\author{
H D Papaconstantinou, A J Marshall, C J Burrell
}

\begin{abstract}
Objective-To assess the feasibility, safety, and clinical impact of diagnostic cardiac catheterisation in a multipurpose laboratory in a district general hospital without cardiac surgery.

Methods-A prospective audit of the first 2000 consecutive cases between September 1992 and March 1997. Unstable patients were referred to a surgical centre for investigation, in line with subsequently published British Cardiac Society (BCS) guidelines, but all other patients requiring cardiac catheterisation were investigated locally and are included in this report. The function of the laboratory was also compatible with the BCS guidelines regarding staffing, operators, equipment, number of cases, and locally available vascular surgery.
\end{abstract}

Results-Of the 2000 cases, 1988 studies were completed (99\%), 1985 (99\%) included coronary angiography, and 1798 $(\mathbf{9 0} \%)$ were performed as day cases. Left main stem disease was present in 157 $(8 \%)$, three vessel disease in $683(34 \%)$, two vessel disease in $387(19 \%)$, single vessel disease in $424(21 \%)$, and normal coronary arteries in $494(25 \%)$. Of the latter, 284 (14\% of the total) had another cardiac diagnosis for which they were investigated (for example, valvar heart disease). Referral for cardiac intervention following catheterisation was made in 1172 of the 2000 cases (intervention rate $59 \%$; catheter:intervention ratio $1.7: 1)$. The interventions performed were coronary artery bypass grafting (CABG) in 736 of the 1172 cases $(63 \%)$, other types of cardiac surgery in $122(10 \%)$, combined CABG and other cardiac surgery in $71(6 \%)$, and percutaneous transluminal coronary angioplasty in $243(21 \%)$. There were two catheter related deaths $(0.1 \%)$, both of which occurred within 24 hours of the procedure, and a further nine major cardiovascular complications with residual morbidity $(0.45 \%)$. These were myocardial infarction in two $(0.1 \%)$, cerebrovascular events in two $(0.1 \%)$, and surgical vascular complications in five $(0.25 \%)$. In addition, there were eight successfully treated, life threatening arrhythmias $(0.4 \%)$.

Conclusions-Diagnostic cardiac catheterisation can be performed safely and successfully in a local hospital. When BCS guidelines are followed, the mortality is similar to published pooled data from regional centres $(0.1 \% v 0.12 \%)$. The high intervention rate indicates a persistent unmet demand in the districts, which will continue to affect surgical and interventional services.

(Heart 1999;81:465-469)

Keywords: cardiac catheterisation; audit; district general hospital; coronary artery disease

A programme for routine diagnostic coronary angiography was developed in Plymouth in September 1992. A prospective audit was initiated from the outset to evaluate the effectiveness and safety of this practice in our hospital. Routine diagnostic cardiac catheterisation, mainly coronary angiography, has since become established in many district general hospitals. The British Cardiac Society (BCS) Council Statement in $1994^{1}$ contained guidelines for the district general hospital cardiac catheter laboratory, which are summarised in table 1 .

This report measures our practice against the BCS guidelines and presents audit data from the first 2000 consecutive cases in terms of morbidity and mortality of the procedure, diagnostic acceptability of the angiograms, and rate of subsequent intervention.

The local district general hospital serves a large district population of approximately 435 000. During this audit, patients were referred to London centres for cardiac intervention and surgery.

\section{Methods}

The aim of the service was to investigate locally all stable cases, while continuing to refer the unstable cases, defined as patients requiring continued intravenous treatment (for example, nitrates, heparin, inotropes) to the surgical centre.

CATHETER LABORATORY STAFFING AND EQUIPMENT

Initially, there were two fully trained invasive cardiologists. Subsequently there were in all

Table 1 The British Cardiac Society Council statement ${ }^{I}$ includes the following recommendations

At least two fully trained operators should be available in each laboratory

Each should be involved with minimum four to five cases a week

The image quality should be as good as that obtained in the tertiary centre laboratories

There should be close links with cardiac surgeons and interventional cardiologists

Training of recognised (junior) post holders is appropriate Emergency cases should be transferred to tertiary centre for investigation, if surgical cover is unavailable on site 
seven experienced (consultant) operators, including six cardiologists (four substantive and two locum appointments) and one radiologist, and 12 trainees (eight cardiology and four radiology). At any one time, the number of experienced consultant operators was no less than two and of cardiology trainees no more than three. Other laboratory staff (cardiac technicians, nurses, and radiographers) underwent training in other centres and in-house.

The $x$ ray equipment was a multipurpose digital Siemens Angiostar with cardiology software, shared with neuroradiology. Data storage was by high quality laser imaged hard copy of single frames selected by the operator, and somewhat lower quality super-VHS videotape.

CLINICAL MANAGEMENT

Precatheter assessment clinics were run by a specially trained cardiac counselling nurse.

All patients undergoing cardiac catheterisation were under the care of a cardiologist who was responsible for the subsequent management decision. Close links were maintained with the referral centres. Initially one and later two cardiologists developed sessional links for coronary angioplasty at the referral centres, and regular joint cardiothoracic clinics operated every two to three months at the local hospital. There was an established comprehensive on-site service for vascular surgery.

\section{AUDIT ORGANISATION}

A single form was designed especially for the purpose of this audit on a two sided A4 size format, recording patient demographics, operator, vascular access, catheter(s) used, angiographic findings, final diagnosis, length of stay, management outcome, and immediate and late complications. Most of the information was recorded on the form by the operator or assistant after each procedure, with subsequent data-for example, late complications or management decisions - added by a registrar. All data were analysed using the Excel 5.0 spreadsheet. Statistical assistance was provided by the local university department.

All immediate and late complications were reported to the catheter laboratory nurse, who acted as the local link to the confidential enquiry of cardiac catheterisation complications (CECCC).

The first 2000 consecutive cases of cardiac catheterisation performed in the multipurpose laboratory have been included in this series. Seventy three cases performed in a mobile unit locally and a similar number referred elsewhere as waiting list initiative activity in 1995 were not included.

DEFINITION OF A COMPLICATION

A complication was defined as any untoward event resulting from the cardiac catheterisation procedure that was life threatening, prolonged hospital stay more than 24 hours, or required a special form of intervention (for example, Doppler compression for a femoral false aneurysm). This is in line with the CECCC concept that a complication is "any untoward event
Table 2 Procedures performed

\begin{tabular}{lrr}
\hline Procedure & $n$ & $\%$ \\
\hline $\begin{array}{l}\text { Left heart catheter and coronary } \\
\text { angiography }\end{array}$ & 1785 & 89.3 \\
$\begin{array}{l}\text { Coronary angiography }{ }^{\star} \text { without left heart } \\
\quad \text { catheter }\end{array}$ & 115 & 5.7 \\
$\begin{array}{l}\text { Left heart catheter without coronary } \\
\quad \text { angiography }\end{array}$ & 4 & 0.2 \\
$\begin{array}{l}\text { Right and left heart catheter and coronary } \\
\quad \text { angiography }\end{array}$ & 85 & 4.3 \\
$\begin{array}{l}\text { Right and left heart catheter without coronary } \\
\quad \text { angiography }\end{array}$ & 2 & 0.1 \\
$\begin{array}{l}\text { Right heart catheter } \\
\text { Abandoned procedure }\end{array}$ & 3 & 0.2 \\
All (adult) diagnostic catheterisations & $\mathbf{2 0 0 0}$ & $\mathbf{1 0 0 . 0}$ \\
\hline
\end{tabular}

${ }^{\star}$ Includes 50 cases with graft angiography.

which jeopardises the patient's life or prolongs the planned hospital stay". ${ }^{2}$

STATISTICS

Confidence intervals (for the overall complication rate) were calculated assuming the Poisson distribution. Comparison between complication rates in different subgroups was by $\chi^{2}$ test (Yates correction).

\section{Results}

DEMOGRAPHICS

Two thousand investigations were performed on 1930 patients, 1409 (73\%) male and 521 female, median age 59 years (range 20 to 86). Most of the cases, 1716 (85.8\%), were studied for suspected coronary artery disease. Other indications included valvar heart disease, adult congenital heart disease, cardiomyopathies, and arrhythmias.

\section{PROCEDURES}

Of the 2000 studies, 1988 (99.4\%) were complete and of at least diagnostic image quality. The 12 incomplete studies included one owing to equipment failure, six procedures abandoned because of bilateral iliac artery occlusion (subsequently performed by an alternative approach), and five owing to failure of selective coronary artery catheterisation.

Almost all procedures $(1985,99.2 \%)$ included coronary (graft) angiography. A complete list by type of procedure is shown in table 2. The femoral approach was used in 1966 cases $(98.3 \%)$, with the brachial (Sones technique) in $27(1.3 \%)$, and the radial in seven $(0.4 \%)$. Our indications for the brachial approach were severe peripheral vascular disease in $13(48.1 \%)$, warfarin treatment or bleeding disorder in $12(44.4 \%)$, severe aortic regurgitation for one, and the intention of early discharge in another. The radial approach was also used mainly because of peripheral vascular disease in four cases $(57.1 \%)$, known coarctation of the aorta in two, and early discharge in one.

\section{CATHETER FINDINGS}

Of the 1988 complete investigations in 1927 patients, $1506(75.8 \%)$ showed coronary artery disease, including 86 with additional structural cardiac disease. Of the remainder, 127 (6.4\%) had valvar heart disease with normal coronary arteries, $71(3.6 \%)$ were investigated for 
Table 3 Diagnoses other than primary coronary artery disease (CAD)

\begin{tabular}{|c|c|c|c|c|}
\hline All cardiac diagnoses other than $C A D$ & & With $C A D$ & $\begin{array}{l}\text { With normal } \\
\text { coronary arteries }\end{array}$ & Total \\
\hline Valvar heart disease & & 77 & 127 & 204 \\
\hline Aortic stenosis & (97) & & & \\
\hline Aortic regurgitation & (32) & & & \\
\hline Mitral stenosis & (5) & & & \\
\hline Mitral regurgitation & $(25)$ & & & \\
\hline Mixed valve disease & $(45)$ & & & \\
\hline Cardiomyopathies & & 1 & 42 & 43 \\
\hline Dilated & (32) & & & \\
\hline Hypertrophic & $(10)$ & & & \\
\hline Arrhythmogenic RV & (1) & & & \\
\hline Congenital heart disease & & 1 & 10 & 11 \\
\hline Atrial septal defect & $(10)$ & & & \\
\hline TGA & (1) & & & \\
\hline Diseases of the aorta and pulmonary vessels & & 3 & 4 & 7 \\
\hline Coarctation & (2) & & & \\
\hline Thoracic aortic aneurysm & (2) & & & \\
\hline Dissection & (1) & & & \\
\hline Pulmonary hypertension & (2) & & & \\
\hline Constrictive pericarditis & & 0 & 1 & 1 \\
\hline Arrhythmias & & 3 & 12 & 15 \\
\hline Pulmonary hypertension & & 1 & 2 & 3 \\
\hline All diagnoses & & 86 & 198 & 284 \\
\hline
\end{tabular}

$\mathrm{RV}$, right ventricular; TGA, transposition of the great arteries.

Table 4 Extent of coronary artery disease, excluding cases with other cardiac disease and normal coronary arteries

\begin{tabular}{lrr}
\hline Extent of coronary disease & \multicolumn{1}{l}{$n$} \\
\hline Left main stem stenosis & 157 & \multicolumn{1}{l}{$\%$} \\
Three vessel disease & 683 & 38.2 \\
Two vessel disease & 387 & 21.6 \\
Single vessel disease & 424 & 23.7 \\
Minimal disease (including post-PTCA) & 86 & 4.8 \\
Normal coronary arteries & 210 & 11.7 \\
Total & $\mathbf{1 7 9 0}$ & $\mathbf{1 0 0 . 0}$ \\
\hline
\end{tabular}

PTCA, percutaneous transluminal coronary angioplasty.

cardiomyopathy, adult congenital heart disease, or arrhythmias, and $284(14.3 \%)$ were essentially normal, including 13 with near a normal appearance after successful angioplasty. The breakdown of all cases with cardiac diagnoses other than coronary artery disease is shown in table 3 . Of all the patients $(n=1506)$ with coronary artery disease, $157(9.5 \%)$ had significant (> 50\%) left main stem disease, 683 $(45.4 \%)$ had significant $(>70 \%)$ three vessel disease, $387(25.7 \%)$ two vessel disease, and $424(28.2 \%)$ single vessel disease (table 4$)$.

MANAGEMENT

In all, 1172 patients went on to an intervention as a result of the investigation, giving an intervention rate of $59 \%$, or a catheter to intervention ratio of $1.7: 1$. Of these, $929(79.3 \%)$ had surgery and $243(20.7 \%)$ coronary angioplasty. The surgical cases included $736(62.8 \%)$ isolated coronary artery bypass grafting (CABG), $71(6.0 \%)$ other cardiac surgery combined with CABG, and $122(10.4 \%)$ other types of cardiac surgery. These included 105 cases of valve surgery, six atrial septal defect repairs, two aortic root and two coarctation repairs, and seven heart transplants. From all the patients referred primarily for valve surgery $(\mathrm{n}=151), 55(36.4 \%)$ required additional coronary artery bypass grafting. The management of patients with coronary artery disease ( $n=1506$ ) grouped according to severity expressed by number of diseased vessels is shown in fig 1 .
COMPLICATIONS

A complete list of complications is shown in table 5. No difference was found between the grade of the operator and the occurrence of any complication $(p=0.63)$. One only of the patients with a complication using the femoral approach was receiving warfarin before the procedure; hence no warfarin hazard can be claimed. In contrast, the Sones technique was associated with a significant increase in total, and particularly vascular, complications $(p=0.03)$. Both deaths occurred in patients with left main stem disease, but statistical tests are difficult to interpret owing to the small numbers.

Death

There were two catheter related deaths. Both patients were middle aged men with left main stem disease. The first had had coronary artery bypass graft surgery 10 years earlier. He presented with unstable angina requiring continuous intravenous treatment and therefore fell outside our guidelines for local investigation. However, he was very keen to be dealt with locally and was investigated by a consultant cardiologist on locum for two weeks from a teaching hospital. There was an occluded dominant right coronary artery and a subtotally occluded left main stem with no patent grafts and well preserved left ventricular function. Unfortunately, he developed cardiogenic shock shortly after the procedure and died six hours later.

The other patient was attending for routine cardiac catheterisation. Severe three vessel disease and a calcified ostial left main stem stenosis were found. Five minutes after completion of the procedure the patient developed chest pain and electrocardiographic changes. Ventricular tachycardia followed, which degenerated rapidly into refractory ventricular fibrillation. He died after a prolonged attempt at resuscitation. A small catheter related dissection flap in the proximal left main stem was found on subsequent study review.

Non-fatal myocardial infarction

There were two cases of non-fatal myocardial infarction. One occurred acutely during catheterisation in a patient with severe calcific aortic stenosis, owing to thromboembolism of the left anterior descending artery. He subsequently underwent successful aortic valve replacement. The other was a non-Q wave infarct diagnosed in a patient with severe three vessel disease, readmitted with chest pain seven hours after the procedure. He also underwent subsequent successful surgery.

\section{Arrhythmias}

Serious arrhythmias occurred in eight cases. Seven had sustained ventricular tachycardia or ventricular fibrillation (VT/VF) during the procedure, requiring external dc cardioversion, one combined with temporary transvenous pacing. One further case developed slow atrial fibrillation requiring temporary pacing, but she was discharged on the same day. 


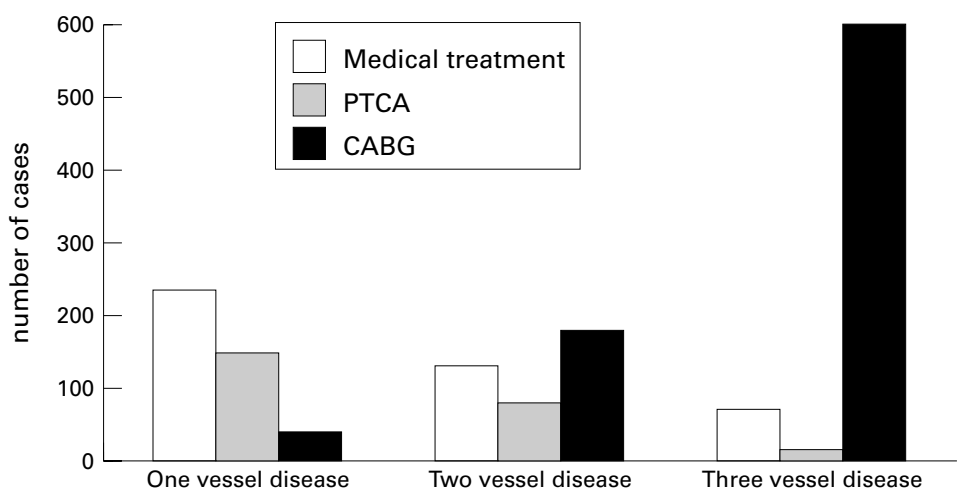

Figure 1 Management decision in the cases with coronary artery disease $(n=1790)$.

Other cardiac complications

One patient developed pulmonary oedema and three had unstable angina requiring active treatment and hospital admission after the procedure. There was one case of intramyocardial injection followed by transient pericarditis. $\mathrm{He}$ was subsequently transferred for urgent CABG surgery because of the severity of his underlying coronary artery disease. There was one significant vasovagal episode which occurred on the ward a few hours after the procedure, associated with bradycardia and hypotension requiring prolongation of hospital admission.

Cerebrovascular events

One stroke resulting from dominant hemisphere thromboembolism occurred in a patient with calcific aortic stenosis. She was subsequently considered unsuitable for cardiac surgery, but made a satisfactory recovery from the stroke. The other suffered a small brainstem event, which required hospital admission for one week, with full neurological recovery. Three patients had postprocedural transient ischaemic attacks, and two were kept in for observation.

Peripheral vascular complications

Surgical-Four had major thromboembolism (three femoral, one brachial) requiring surgery and one had a femoral pseudoaneurysm, also requiring surgical repair. There was significant residual morbidity in only one patient with iliofemoral thromboembolism and severe preexisting peripheral vascular disease.

Non-surgical-Four patients had femoral pseudoaneurysms closed as outpatients, using external, Doppler guided compression. There were four large groin haematomas. Two resulted in deep venous thrombosis, which was treated medically. One patient had a rebleed from the femoral puncture site on the ward requiring further compression and overnight observation. One patient developed infected cellulitis over the brachial cutdown in the right antecubital fossa, which required readmission for intravenous antibiotics.

\section{Contrast agent hypersensitivity}

There were two allergic reactions to the radioopaque contrast medium, one with urticaria and the other with pruritus and mild bronchospasm. They were kept in hospital for 24 hours as a precaution.

\section{Pseudocomplications}

One patient suffered a VT/VF arrest while waiting for the procedure in the catheter laboratory. He was successfully resuscitated and had the procedure performed uneventfully on the same day.

\section{Discussion}

Diagnostic cardiac catheterisation is an invasive investigation with potential for complications, including death. Our primary aim was to establish whether it could be performed safely in a local general hospital without cardiac surgery on site or nearby. We aimed to collect complete data prospectively to avoid the bias inherent in retrospective studies, particularly where data are collected from subsidiary source records such as theatre logbooks.

Improvements in technique and technology have reduced the morbidity of routine diagnostic coronary angiography, but occasional mortality seems to be unavoidable. ${ }^{3}$ Most series report a mortality of between $0.1 \%$ and

Table 5 All complications by type, with frequency seen in other reports for comparison

\begin{tabular}{|c|c|c|c|c|c|c|c|}
\hline \multirow[b]{2}{*}{ Complication } & & \multicolumn{3}{|c|}{ This series $(n=2000)$} & \multirow{2}{*}{$\begin{array}{l}C A S S^{7} \\
\%\end{array}$} & \multirow{2}{*}{$\begin{array}{l}S C A^{4} \\
\%\end{array}$} & \multirow{2}{*}{$\begin{array}{l}C E C C^{2} \\
\%\end{array}$} \\
\hline & & $n$ & $\%$ & Admitted & & & \\
\hline Death & & 2 & 0.10 & & 0.20 & 0.14 & 0.12 \\
\hline Non-fatal myocardial infarction & & 2 & 0.10 & 2 & 0.25 & 0.07 & 0.16 \\
\hline Arrhythmia & & 8 & 0.40 & 1 & & 0.56 & 0.22 \\
\hline Other cardiac complications & & 5 & 0.25 & & & & \\
\hline Acute left ventricular failure & (1) & & & 1 & & & \\
\hline Unstable angina pectoris & (2) & & & 2 & & & \\
\hline Intra- or transmyocardial injection & (1) & & & 1 & & & \\
\hline Vasovagal reaction & (1) & & & 1 & & & \\
\hline Cerebrovascular event & & 5 & 0.25 & 4 & & 0.07 & 0.02 \\
\hline Stroke & (2) & & & & & & \\
\hline Transient ischaemic attack & (3) & & & & & & \\
\hline Surgical vascular complication & & 5 & 0.25 & 5 & & & 0.19 \\
\hline Non-surgical vascular complication & & 10 & 0.50 & 5 & & & \\
\hline Femoral false aneurysm (compressed) & $(4)$ & & & & & & \\
\hline Haematoma (large) & (2) & & & & & & \\
\hline Haematoma and deep vein thrombosis & (2) & & & & & & \\
\hline Rebleed & (1) & & & & & & \\
\hline Entry site infection & (1) & & & & & & \\
\hline Contrast reaction & & 2 & 0.10 & 1 & & & \\
\hline Pseudocomplication & & - & & & & & \\
\hline Preprocedure cardiac arrest & (1) & & & & & & \\
\hline All complications & & 39 & 1.95 & 23 & & & 0.82 \\
\hline
\end{tabular}


$0.2 \% .^{24-9}$ It is related to the severity of the underlying disease and functional class of the patient, rather than the technique used or the experience of the operator. ${ }^{45}$

To minimise risk, we decided to select only stable patients for local investigation in an attempt to avoid preventable mortality and on the basis that almost all persistently unstable patients will proceed to intervention not available at the local hospital. It has been suggested that fatality may be avoided by immediate surgery, ${ }^{10-12}$ which has been an argument against undertaking such investigations routinely without local surgical cover. However, the $0.1 \%$ mortality in this series is in line with previous reports ${ }^{4-8}$ and the recent pooled national United Kingdom data derived from regional centres with on-site or nearby surgical cover $\left(0.12 \%\right.$, CECCC Registry $\left.{ }^{2}\right)$.

Reporting of non-fatal complications is more variable, and rates depend partly on differences in definitions and in case mix. We report a complication rate higher than that in the CECCC report ${ }^{2}(1.9 \%$ with $95 \%$ confidence interval (CI) 1.3 to $2.5, v 0.8 \%$ with $95 \%$ CI 0.7 to 0.9 ), but similar to recent data from another UK centre ${ }^{9}$ (4.4\% with $95 \%$ CI 3.1 to $5.8)$. The increased rate of vascular complications in the non-femoral group may be correlated with the high proportion of patients with severe peripheral vascular disease in that group $(\sim 50 \%)$. We report rates of allergic reaction similar to or lower than other UK centres $(0.1 \% v 0.2 \%))^{29}$ The widespread use of nonionic contrast media thus seems unlikely to entirely explain the lower complication rate in the CECCC data, as previously suggested. ${ }^{2}$

Supervised training was maintained throughout this audit. This did not seem to have an adverse effect on safety, and each trainee performed a reasonable number of procedures. It should be possible to meet the needs of cardiology trainees through integrated local hospital/regional centre training programmes.

The unmet demand for investigation and intervention, combined with the high prevalence of coronary artery disease in our health district (age standardised death rates from coronary artery disease in the highest range in the country at 55.4 per $100000^{13}$ ), ensured a high rate of intervention initially. The overall catheter to intervention ratio was $1.7: 1$, but it increased across the series from 1.5:1 (first 250 cases $^{14}$ ) to 1.9:1 (last 250 cases) as this demand has been slowly addressed. Thus a hospital developing a new service for cardiac catheterisation can expect a high initial intervention rate and this may apply particularly to geographically remote centres.

Videotape is a suboptimal storage medium and previous studies have reported poor quality coronary angiograms obtained in dis- trict general hospitals using this method. ${ }^{15}$ Fortunately, none of the diagnostic catheter studies we produced locally was actually repeated at the surgical centre because of poor quality alone. The parallel use of high quality, laser printed hard copy of single frames and close liaison with the referral centre was helpful in this regard.

\section{CONCLUSIONS}

We believe that diagnostic cardiac catheterisation can be performed safely and effectively in a local hospital without on-site cardiac surgery. However, from the lessons learned during this audit process, we believe that there are certain important features that will predict successful programmes. These include a consultant based and consultant led service, the establishment of close relations with the surgical centre and the continued referral of unstable patients, the use of a high quality digital storage medium, and the development of a well trained, motivated, and enthusiastic local catheter laboratory team.

1 British Cardiac Society Council Statement. Strategic planning for cardiac services and the internal market: role of catheterisation laboratories in district general hospitals. Br Heart f 1994;71:110-12.

2 De Bono D, on behalf of Joint Audit Committee of the British Cardiac Society and the Royal College of Physicians of London. Complications of cardiac catheterisation: results from 34041 patients in the UK confidential enquiry into cardiac catheter complications (CECCC). Br Heart $\mathcal{f}$ 1993;70:297-300.

3 Judkins MP, Gander MP. Prevention of complications of coronary arteriography. Circulation 1974;49:599-602.

4 Kennedy JW, chairman and the Registry Committee of the Society For Cardiac Angiography. Symposium on catheterisation complications. Complications associated with cardiac catheterisation and angiography. Cathet Cardiovasc Diagn 1982;8:5-11.

5 Kennedy JW, Baxley WA, Bunnell IL, et al. Mortality related to cardiac catheterisation and angiography. Cathet Cardiovasc Diagn 1982;8:323-40.

6 Bourassa MG, Noble J. Complication rate of coronary arteriography: a review of 5250 cases studied by a percutaneous femoral technique. Circulation 1976;53:106-14

7 Davis K, Kennedy JW, Kemp HG, et al. Complications of coronary arteriography from the collaborative study of coronary artery surgery (CASS). Circulation 1979;59:110512 .

8 Noto TJ, Johnson LW, Krone R, et al, and the Registry Committee of the Society for Cardiac Angiography and Interventions. Cardiac catheterisation 1990: a report of the Registry of the Society for Cardiac Angiography and Interventions (SCA\&I). Cathet Cardiovasc Diagn 1991;24:7583.

9 Oldroyd KG, Phadke KV, Phillips R, et al. Cardiac catheterisation by the Judkins technique as an outpatient procedure. BMF 1989;298:875-6.

10 Stewart JT, Gray HH, Ward DE, et al. Major complications of coronary arteriography: the place of cardiac surgery. $\mathrm{Br}$ Heart $\mathcal{F}$ 1990;63:74-7.

11 Millane TA, Bridgewater B, Jones MT, et al. Diagnostic cardiac catheterisation and emergency cardiac surgery [abstract]. Br Heart f 1995;73 (suppl): P76.

12 Kovac J, De Bono DP. Cardiac catheter complications related to left main stem stenosis [abstract]. Br Heart $\mathcal{F}$ 1995;73(suppl):P76.

13 OPCS. Mortality files 1993, 1994, 1995. London: Office of Population Censuses and Surveys.

14 Burrell CJ, Papaconstantinou H, Cowley ML, et al. Multi-purpose cardiac catheterisation laboratory in a district general hospital: audit of the first 250 cases [abstract]. Br Heart $\mathcal{F} 1994 ; 71$ (suppl):S322

15 Ranjadayalan K, Mills PG, Sprigings DC, et al. Coronary arteriography in a district general hospital: feasibility, safety and diagnostic accuracy. BMF 1990;300:777-80. 